\title{
Electrochemical Quantification of Piperine in Black Pepper
}

Yuanzhe Wang, Lifu Chen, Korbua Chaisiwamongkhol, Richard G Compton*

Department of Chemistry, Physical and Theoretical Chemistry Laboratory, Oxford

University, South Parks Road, Oxford OX1 3QZ, UK

*Corresponding author

Email: richard.compton@chem.ox.ac.uk

Phone: +44 (0) $1865275957 \quad$ Fax: +44 (0) 1865275410

Email addresses of co-authors:

yuanzhe.wang@wadham.ox.ac.uk

lifu.chen@ chem.ox.ac.uk

korbua.chaisiwamongkhol@wolfson.ox.ac.uk

To be submitted to:

Food Chemistry 


\section{$1 \quad$ Abstract}

2 A simple, rapid method of the detection of piperine in black pepper is reported using a voltammetric

$7 \quad$ Keywords

8 Piperine

$9 \quad$ Semi-circular potential sweep voltammetry

$$
\text { Detection in black pepper }
$$

\section{Introduction} sensor based on a glassy carbon electrode (GC) with analysis following a short one-step extraction using ethanol. The method is based on a novel potential sweep designed to maximise signal sizes and shown with context of the present analytical challenge to be essential for gathering data allowing the construction of a linear calibration curve for the analysis in the relevant range namely $0.25-5.0 \mathrm{mM}$.

Black pepper (Piper nigrum), known as the "king of spice", is one of the most widely consumed spices in the world. The two main components controlling the quality of black pepper are first piperine, a naturally occurring alkaloid which principally contributes to its distinct pungency and second the volatile oils responsible for its aroma (Parthasarathy, Chempakam \& Zachariah, 2008). As the most abundant pungent component constituting $98 \%$ of the total alkaloids in pepper (Hirasa \& Takemasa, 1998), the piperine content has been previously used for a measure of total pungency in black pepper (Parthasarathy et al., 2008). The percentage of piperine content was reported to vary from $2.0 \%$ to $9.0 \%$ (w/w) of black pepper among different cultivars, depending on climate, growing conditions harvesting process and the method of extractions (Peter, 2001; Gorgani, Mohammadi, Najafpour \& Nikzad, 2017).

Piperine has been shown to possess diverse biological and therapeutic properties that are beneficial for human health. It exhibits anti-microbial (Zarai, Boujelbene, Ben Salem, Gargouri \& Sayari, 2013), anti-depressant (Mao, Huang, Zhong, Xian \& Ip, 2014), anti-tumour (Doucette, Hilchie, Liwski \& Hoskin, 2013) and high anti-oxidant activities (Vijayakumar, Surya \& Nalini, 2004). It also exhibits a hepatoprotective property (Sabina, Souriyan, Jackline \& Rasool, 2010) and immuno-modulatory 
properties (Sunila \& Kuttan, 2004), and can act as an anti-inflammatory for the treatment of asthma (Kim \& Lee, 2009). The inhibitory effect of piperine in breast cancer (Doucette et al., 2013) and its possible cytotoxic and anti-HIV activity (Shimada et al., 2014) have also been reported. In addition, Piperine can enhance the bioavailability of a variety of drugs, such as antibiotics like norfloxacin (Janakiraman \& Manavalan, 2008) and rifampicin (Balakrishnan, Varma \& Chatterji, 2001), as well as conventional herbal medicines like curcumin (Mueller \& Hingst, 2013).

Owing to the importance of piperine in culinary use and biomedical applications, a simple and accurate determination of piperine is required for both the food industry and for pharmaceutical use. Several analytical techniques for quantifying the amount of piperine extracted from black pepper have been reported. The colorimetric method and Kjeldahl method rely on chemical reactions of piperine but are thought to overestimate the piperine contents due to the presence of other compounds in black pepper with similar functional groups (Gorgani et al., 2017). Gas chromatography-mass spectrometry (GC-MS), ultra-visible spectrophotometry (UV-Vis), and high-performance liquid chromatography (HPLC) offer good specificity and sensitivity (Noyer, Fayet, Pouliquen-Sonaglia, Guerere \& Lesgard, 1999). In particular HPLC, provides better accuracy and hence becomes the most commonly used technique in the detection of piperine (Hirasa \& Takemasa, 1998). However, these methods require the use of expensive instruments and elaborate laboratory conditions to perform the analysis. Also, the complicated sample preparation steps can only be conducted by well-trained personnel and consume relatively long time rendering it difficult for frequent commercial use.

Electrochemistry as a qualitative and quantitative analytical technique can overcome the above mentioned problems due to the simplicity of experimental set-ups, high sensitivity and cost effectiveness. There are several reports of piperine detection using electrochemistry. Electrochemical detection (ECD) for HPLC was used with a detection limit obtained of the same order of that in UV detection (Ternes \& Krause, 2002). Electrochemical Impedance Spectroscopy (EIS) using a surfacepolarity controlled sensor with chemometric treatment was also proposed to discriminate piperine from other pungent substances (Ju, Hayama, Hayashi \& Toko, 2003). These methods again involve relatively complicated treatments or analysis and no detection range was reported for applied use. A voltammetric 
study on a glassy carbon employing cyclic voltammetry and square wave voltammetry was reported (Jain \& Verma, 2017), following an elaborate extraction process. Among the electrochemical techniques, cyclic voltammetry is the most frequently used as it provides abundant information of the studied redox system notably analyte concentrations, rate constants and diffusion coefficients. It conventionally employs triangular potential waveform where the scan rate is constant throughout the entire voltammetric sweep (Bard \& Faulkner, 2001; Compton \& Banks, 2018).

However, recently, a method performing the voltammetry using a semi-circular potential wave has been proposed for electroanalysis (Amin, Uchida, Kätelhön \& Compton, 2019; Uchida, Kätelhön \& Compton, 2018), where an infinite scan rate is generated at the midpoint of applied potential window and relatively low scan rates at other parts. As a result, the current response is significantly amplified at the midpoint and suppressed at other regions. The semi-circular potential sweep voltammetry offers significant improvement of the sensitivity of voltammetric measurement especially for electroanalytical purposes (Amin et al., 2019); this is investigated in the present paper, building on the work of Jain and Verma (Jain \& Verma, 2017), re-examining and correcting the mechanism and proposing a much simplified extraction procedure. In particular, the use of the more sensitive semi-circular potential sweep voltammetry and baseline correction facilitated the latter by allowing an optimal extraction solvent system to be employed.

In this work, we investigate the voltammetric and chronoamperometric behaviours of piperine and propose a reduction mechanism at both macro-and micro-electrodes. We also report an electrochemical method for detection of piperine employing the voltammetry in combination with a semi-circular potential sweep at a glassy carbon (GC) electrode. In addition, we study real samples and suggest a simple one-step sample preparation, noting that the reductive aqueous voltammetry of piperine is not interfered by any of the inorganic ions $\mathrm{Na}^{+}, \mathrm{K}^{+}, \mathrm{SO}_{4}{ }^{2-}, \mathrm{Ca}^{2+}, \mathrm{NH}_{4}{ }^{+}, \mathrm{CO}_{3}{ }^{2-}$, or the molecules glucose, fructose, vanillin, capsaicin cinnemaldehyde (Jain \& Verma, 2017). Finally, we demonstrate the applicability of the developed methods for detection and quantification of piperine in black peppercorn samples. 


\section{Materials and methods}

82

\subsection{Chemicals and reagents}

All chemicals were of analytical grade and were used received without any further purification. Piperine $(\geq 97 \%)$ was purchased from Aldrich and sodium hydroxide (97\%) was purchased from Honeywell. All solutions were prepared with ethanol (Sigma-Aldrich, $\geq 99.8 \%$ ) or deionised water at a resistivity of 18.2 $\mathrm{M} \Omega \mathrm{cm}$ at $298 \mathrm{~K}$ (Millipore, MA, USA). Sainsbury's "Fairtrade Organic Whole Black Peppercorns" were purchased from Sainsbury's (Oxford, UK). The phosphate-buffered saline (PBS, $1 \times, \mathrm{pH}=7.4)$ was prepared using sodium chloride $(137 \mathrm{mM}$, Sigma-Aldrich, $\geq 99.5 \%)$, potassium chloride (2.7 mM, Sigma-Aldrich, $\geq 99.0 \%$ ), Sodium phosphate dibasic (10 mM, Sigma-Aldrich, $\geq 99.0 \%$ ), and potassium phosphate monobasic (1.8 mM, Sigma, $\geq 99.0 \%)$.

\subsection{Electrochemical instrumentation and methods}

All electrochemical experiments were conducted in a Faraday cage at $298 \mathrm{~K}$ using a standard threeelectrode system. The cyclic voltammetric measurements and the chronoamperometry were performed using a $\mu$ Autolab II potentiostat (Metrohm-Autolab BV, Netherlands).

For cyclic voltammetry, a glassy carbon (GC) macroelectrode (calibrated as $2.99 \mathrm{~mm}$ diameter) or a carbon fibre microdisc electrode (ALS Co. Ltd., Japan) was used as the working electrode, a saturated calomel electrode (SCE, ALS distributed by BASi Inc., Japan.) as the reference electrode and a graphite rod as the counter electrode. The radius of carbon fibre microdisc was calibrated electrochemically as $21.3 \mu \mathrm{m}$ by analysing the steady state voltammetry of $1.0 \mathrm{mM}$ hexaamineruthenium(III) chloride in 0.1 $\mathrm{M} \mathrm{KCl}$ aqueous solution (Figure S1), using a diffusion coefficient for $\left[\mathrm{Ru}\left(\mathrm{NH}_{3}\right)_{6}\right]^{3+}$ of $8.43 \times 10^{-6} \mathrm{~cm}^{2} \mathrm{~s}^{-1}$ at $298 \mathrm{~K}$ (Wang, Limon-Petersen \& Compton, 2011). The glassy carbon (GC) macroelectrode and carbon fibre microdisc electrode were polished onto the soft lapping pads (Buehler, UK) before each voltammetric experiment, using alumina with particle size of 1.0, 0.3 and $0.05 \mu \mathrm{m}$, (Buehler, IL, UK), in descending order of size, followed by polishing on a blank pad, rinsing with deionised water and drying with nitrogen. For chronoamperometry, the same carbon fibre microdisc was used as the working electrode, the same reference electrode and counter electrode were used as above. 
Semi-circular potential wave sweep voltammetry was carried out with a computer-controlled in-lab built potentiostat, the configuration and merits of which were described previously (Batchelor-Mcauley et al., 2015; Amin et al., 2019). The in-lab potentiostat ensures the low-noise measurements with signal sampled at a stream rate of $100 \mathrm{kHz}$. It was controlled by script written in Python 3.5 to generate required potential waveform. Modification of commercial potentiostat would likely require the addition of a suitable waveform generator. The glassy carbon (GC) as above was used as the working electrode, which was held at the start potential of the chosen potential window for $3 \mathrm{~s}$ prior to applying the potential wave, with the same reference electrode and counter electrode also used as above.

\subsection{Sample Preparation}

To conduct the analysis of real samples, piperine was extracted from black peppercorns. The black peppercorns were ground finely by the grinder provided as part of the jar containing the sample. $0.5 \mathrm{~g}$ of the ground sample was placed into a sampling vial, and $5 \mathrm{~mL}$ ethanol was added into the vial, followed by vigorously shaking for 1 minute. The vial was then sealed and placed in an ultrasonic bath (FB15050, Fisher Scientific, 50/60 Hz, $80 \mathrm{~W}$, Germany) for 5-15 minutes of sonication or left to settle for 1-120 minutes. The supernatant was collected for further analysis.

\subsection{Analytical Procedures}

A piperine stock solution of $15.0 \mathrm{mM}$ was prepared before diluted with the mixture of $60 \%$ ethanol + $40 \% \mathrm{PBS}$ at $\mathrm{pH}=7.4$ to concentration $10.0,5.0,4.0,3.0,2.5,2.0,1.5,1.0,0.75,0.5,0.25 \mathrm{mM}$ as working solutions. This solvent was chosen due to the low solubility of piperine in aqueous solution. electrode was first placed in a solution containing $1 \mathrm{mM}$ piperine dissolved in a mixture of $60 \%$ ethanol $+40 \% \mathrm{PBS}$ at $\mathrm{pH}=7.4$. Prior to the measurements, the solutions were bubbled with $\mathrm{N}_{2}$ for 10 minutes to remove the dissolved oxygen. The potential window was chosen to be 0 to $-2.5 \mathrm{~V}$ and the voltammograms were recorded at different scan rates $\left(25,50,100,150,200,400 \mathrm{mVs}^{-1}\right)$. Analogous experiments were then conducted under air-saturated conditions. To investigate the electrochemical 
process under basic conditions, $1 \mathrm{mM}$ piperine solution containing $60 \%$ ethanol $+40 \% \mathrm{NaOH}(0.1 \mathrm{M}$, 1.0 $\mathrm{M}$ and $2.0 \mathrm{M}$ ) was used with $\mathrm{N}_{2}$ bubbling as above. The potential window was chosen as -1.2 to $1.8 \mathrm{~V}$. The voltammograms were recorded at a scan rate of $1.0 \mathrm{Vs}^{-1}$.

For the chronoamperometric experiments using a carbon fibre microdisc as the working electrode, the electrode was placed in a solution containing 5, 10, $15 \mathrm{mM}$ piperine in $60 \%$ ethanol $+40 \%$ PBS or $60 \%$ ethanol $+40 \% \mathrm{NaOH}(0.1 \mathrm{M}, 1.0 \mathrm{M}$ and $2.0 \mathrm{M})$ mixture under degassed conditions as above. Chronoamperometric transients were recorded with a sample time of $0.002 \mathrm{~s}$. The potential was stepped from $0 \mathrm{~V}$ to the reductive potential after the pre-equilibration for $3 \mathrm{~s}$. The current was measured for $5 \mathrm{~s}$, and data points before $20 \mathrm{~ms}$ related to non-Cottrellian behaviour as a result of considerable doublelayer charging were removed (Xiong, Aldous, Henstridge \& Compton, 2012).

For real sample analysis using the glassy carbon (GC) electrode, the extracts obtained as above but with PBS added were diluted with $60 \%$ ethanol $+40 \%$ PBS (dilution factor was 2 ) such that the voltammetric responses fell within the linear detection range. The cyclic voltammetric and semi-circular potential wave sweep voltammetric responses were recorded under air-saturated conditions at a scan rate of 100 (cyclic voltammetry) or $50 \mathrm{mVs}^{-1}$ (semi-circular potential wave sweep voltammetry).

\section{Results and Discussion}

In the following sections, first, the electrochemical behaviour of piperine (structure shown in Figure 1(A) inset) on glassy carbon (GC) macroelectrodes and carbon fibre microdisc electrodes are studied to investigate the possible mechanism of piperine reduction. Second, the analytical responses using cyclic voltammetry and semi-circular sweep voltammetry for the detection of piperine at GC macroelectrode are studied. Finally, an optimised extraction procedure of black pepper is developed and both methods are used to quantify piperine contents in real samples.

\subsection{Electrochemical Study of Piperine}

\subsubsection{Effect of Voltage Scan Rate}


We looked first at the cyclic voltammetric signal of piperine at a glassy carbon (GC) macroelectrode.

159 Figure 1 (A) depicts the voltammetric responses of piperine at GC electrode in degassed 60\% ethanol

$160+40 \%$ PBS solution in presence and absence of $1 \mathrm{mM}$ piperine, at a scan rate of $100 \mathrm{mVs}^{-1}$. An

161 electrochemically irreversible reduction peak of piperine can be observed at $-1.7 \mathrm{~V}$ vs. SCE. The effect

162 of scan rates on the reduction peak currents of $1.0 \mathrm{mM}$ piperine at GC macroelectrode was then studied.
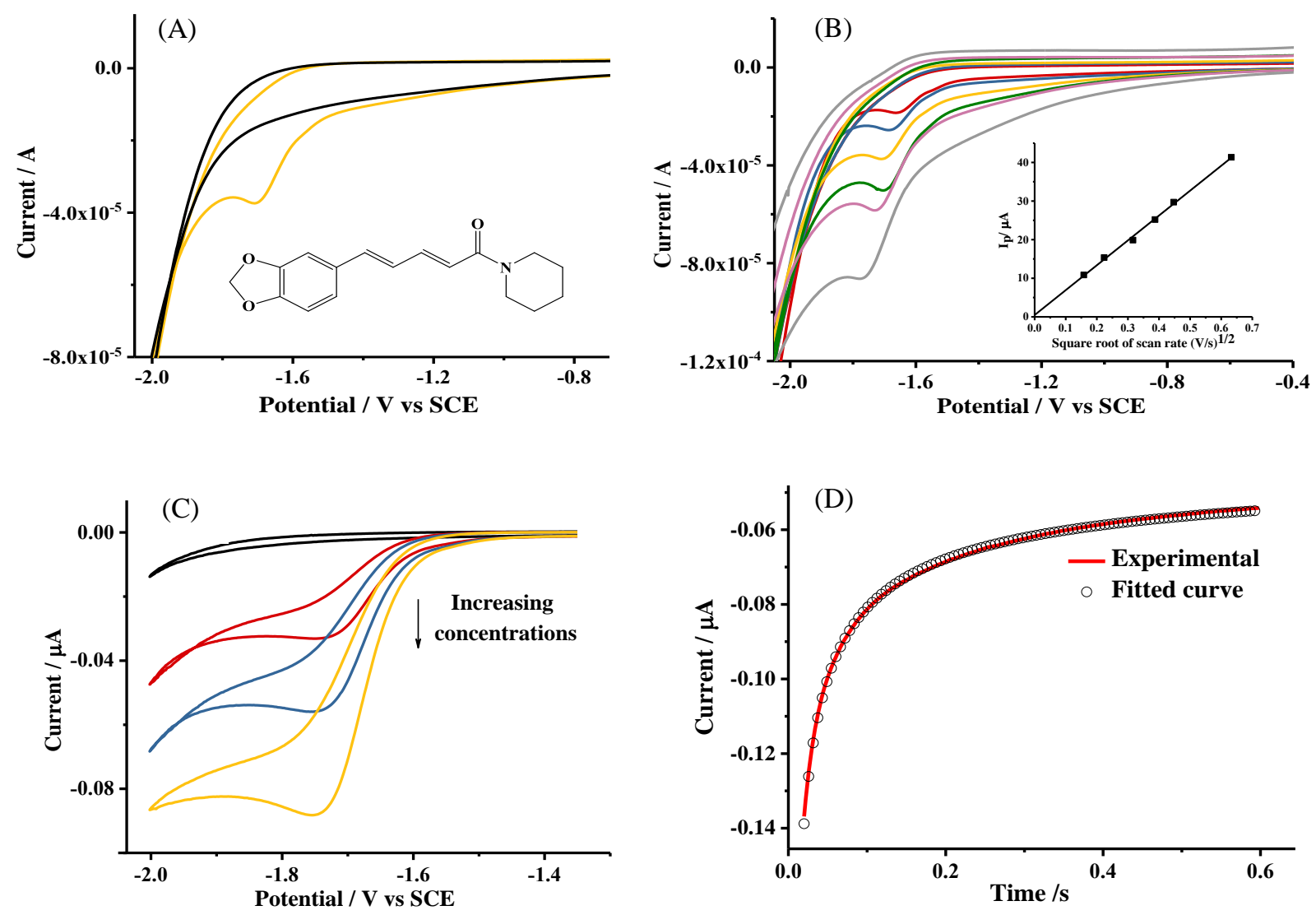

(E)<smiles>[R14]C=CC=CC([R1])=O</smiles><smiles>[R4]C=CCCC([R1])=O</smiles>

Figure 1. Electrochemical study of piperine in $60 \mathrm{EtOH}: 40$ PBS under degassed conditions: cyclic voltammetric responses at glassy carbon (GC) macroelectrode of (A) background (black line) and $1 \mathrm{mM}$ piperine (yellow line) at a scan rate of $100 \mathrm{mVs}^{-1}$ (B) $1 \mathrm{mM}$ piperine reduction at varying scan rates of $25 \mathrm{mVs}^{-1}, 50 \mathrm{mVs}^{-1}, 100 \mathrm{mVs}^{-1}$, $150 \mathrm{mVs}^{-1}, 200 \mathrm{mVs}^{-1}, 400 \mathrm{mVs}^{-1}$; at a carbon fibre microdisc electrode of (C) $0 \mathrm{mM}$ and $5 \mathrm{mM}, 10 \mathrm{mM}, 15 \mathrm{mM}$ of piperine at a scan rate of $100 \mathrm{mV} / \mathrm{s}$. (D) Chronoamperometric response of $10 \mathrm{mM}$ piperine with solvent subtracted (red solid line) at carbon fibre microdisc, and corresponding line of best fit (black circles) derived from the Shoup and Szabo equation. (E) Possible two-step reaction mechanism of piperine reduction. Inset of (A) chemical structure of piperine (B) plot of peak current versus square root of scan rate. 
172 Cyclic voltammograms were recorded in $60 \%$ ethanol $+40 \%$ PBS at the scan rates in the range of 25 173 to $400 \mathrm{mVs}^{-1}$ (Figure 1(B)). As shown in Figure 1(B) inset, the peak current (Ip) was found to exhibit a 174 linear relationship with the square root of scan rates from 25 to $400 \mathrm{mVs}^{-1}$, suggesting the reduction of 175 piperine is a diffusion-controlled electrochemical process. This was further confirmed by the linearity observed in the plot of $\log \left(\mathrm{I}_{\mathrm{p}}\right)$ versus $\log (\mathrm{v})$ (Figure $\mathrm{S} 2$ ).

\subsubsection{Measurement of Number of Electrons Transferred and the Diffusion Coefficient}

179 In order to further investigate this diffusion-controlled reduction process and, in particular, to determine 180 the diffusion coefficient of piperine in the studied system, the chronoamperometric responses of varying 181 concentrations of piperine were recorded at a carbon fibre microdisc electrode. In the potential step 182 chronoamperometric experiment, the potential applied to the microdisc electrode was stepped from 0 $183 \mathrm{~V}$, where no current flows, to an overpotential which ensures the reduction occurs at a rate controlled by mass transport. The overpotential we used for the chronoamperometry was determined from the cyclic voltammetry as -1.8 V vs. SCE to ensure diffusion control. (Figure 1(C) Section 3.1.1) measurement:

$$
I=-4 n F \operatorname{Dcrf}(\tau)
$$

where

$$
f(\tau)=0.7854+0.8863 \tau^{-1 / 2}+0.2146 \exp \left(-0.7823 \tau^{-1 / 2}\right)
$$

$$
\tau=\frac{4 D t}{r^{2}}
$$


where $I$ is the current, $F$ is the Faraday constant, $n$ is the number of electrons transferred, $D$ and $c$ are respectively the diffusion coefficient and the concentration of the electroactive species, $r$ is the radius of the microdisc, and $\mathrm{t}$ is the time. The current dependency on diffusion coefficient changes from $I \propto$ $\sqrt{D}$ at short time to $I \propto D$ at long time, whilst the current is proportional $(n c)$ to at all times. Thus for a given known concentration, $n$ and $D$ can both be deduced by fitting experimental transient with the Shoup-Szabo equation as used effectively in several previous studies (Banks et al., 2005; Silvester, Wain, Aldous, Hardacre \& Compton, 2006; Paddon, Silvester, Bhatti, Donohoe \& Compton, 2007). The time interval of $0.02-0.6 \mathrm{~s}$ of the chronoamperometric transient was analysed to obtain an accurate fit characterising the diffusional process, without including the data points describing the nonCottrellian behaviour controlled by double layer charging before $20 \mathrm{~ms}$ and an excessive contribution from the steady state current at long time (Xiong et al., 2012). The fitting was achieved via ORIGIN 2017, where by inputting the known values of $r$ and $c$, the software iterated through the values of $n$ and $D$ until an optimised fit was obtained. It is noted that since the chronoamperometric experiments were performed at the high negative potential at $-1.8 \mathrm{~V}$ vs. SCE, the solvent transient originating from the redox reaction of solvent and the double layer charging can 'obscure' the chronoamperometric responses of interest (Compton \& Banks, 2018). Consequently, the transient of solvent without the addition of piperine was subtracted from measured chronoamperometric transient before fitting with the theoretical curve for a more accurate evaluation of $n$ and $D$.

Figure 1(D) (section 3.1.1) shows the experimental potential step chronoamperometric curve of 10 $\mathrm{mM}$ piperine at carbon fibre microdisc electrode and the corresponding fit. The chronoamperometric signal again indicates the process is typically diffusion-controlled. Chronoamperometric measurements of varying concentrations of $5,15 \mathrm{mM}$ piperine were also conducted and the same method of analysis was used (Figure S3). The average of results given by the fitting curves gave diffusion coefficient of $(4.9 \pm 0.5) \times 10^{-6} \mathrm{~cm}^{2} \mathrm{~s}^{-1}$ (at $298 \mathrm{~K}$ ), with the number of electrons transferred found to correspond to 1 (Table S1). 


\subsubsection{Possible Reaction Mechanism}

Having identified the electrochemical process occurring at the microelectrode, we next compared it with the results obtained from the glassy carbon (GC) macroelectrode (Figure 1(B) Section 3.1.1). The peak current, $\mathrm{I}_{\text {Irrev }}$, was calculated for an irreversible, one-electron electrochemical reduction using the parameters ( $n$ and $D$ ) deduced from the chronoamperometry via the Randles-Sevcik equation:

$$
I_{p}=0.496 \sqrt{n^{\prime}+a_{n^{\prime}+1}} n F A[A]_{b u l k} \sqrt{\frac{F D v}{R T}}
$$

where $n^{\prime}$ is the number of electrons transferred before the rate determining electron transfer, $n$ is the total number of electrons transferred, $\alpha$ is the electron transfer coefficient, taken as $0.5, F$ is the Faraday constant, $A$ is the electrode area, $[A]_{b u l k}$ is the bulk concentration, $D$ is the diffusion coefficient equal to $4.9 \times 10^{-6} \mathrm{~cm}^{2} \mathrm{~s}^{-1}$ measured in section 3.2.2, $v$ is the scan rate, $R$ is the gas constant and $T$ is the temperature of $298 \mathrm{~K}$. Assuming $n^{\prime}=0, n$ for the electron transfer at macroelectrode can also be determined using the peak currents $\left(\mathrm{I}_{\text {Expt }}\right)$ measured at varying scan rates using cyclic voltammetry as described in section

\subsection{1 (see Table S2).}

Comparing the $\mathrm{I}_{\text {Irrev }}$ values calculated assuming a one-electron process with $\mathrm{I}_{\text {Expt }}$, which characterises the electrochemical process on macroelectrode, the $\mathrm{I}_{\text {Expt }}$ values are ca. twice as large as the values of Irrev. It can be inferred that in contrast to the one electron transfer process suggested by the microelectrode chronoamperometric responses, the reduction at a GC macroelectrode involves a twoelectron transfer.

The chemical reduction of piperine under various conditions has been reported (da Silva, Mota, Bieber \& Navarro, 2006; Gallagher, Shimmon \& McDonagh, 2012; Pedersen et al., 2009). Either of the two carbon-carbon double bonds has been generally suggested to undergo hydrogenation in methanol (or ethanol) with a catalyst or mediator (Gallagher et al., 2012), although reduction leading to a $\alpha, \beta$ unsaturated amide was also claimed (da Silva et al., 2006; Pedersen et al., 2009). For the electrochemical reduction as discussed above, a possible mechanism explaining the difference between the micro- and macro-electrode voltammetry is shown in Figure 1(E), Section 3.1.1. The one-electron transfer 
electrochemical reduction generates the carbanion species in the first step, followed by protonation and

244 further one-electron transfer, resulting in the formation of an unsaturated ketone. From this mechanism,

245 the discrepancy between the electrochemical processes at macroelectrode and microelectrode can be rationalised since at carbon fibre microdisc electrode, "divergent/convergent diffusion" occurs, reflecting the significantly more efficient mass transport (Compton \& Banks, 2018). The intermediate, carbanion, thus may diffuse away from the electrode surface before the chemical step needed for the second electron transfer can occur. In contrast at a glassy carbon (GC) macroelectrode, the diffusion is linear, so that the loss of the carbanion intermediate is much reduced, allowing both steps to occur and an overall transfer of two electrons to take place.

In order to attempt possibly to slow the proton transfer step, further experiments were made at high pH. However, cyclic voltammetric measurements at GC macroelectrode and chronoamperometric measurements at carbon fibre microdisc conducted under high $\mathrm{pH}$ conditions in $60 \%$ ethanol $+40 \%$ aqueous solution of $[\mathrm{NaOH}]=0.1,1.0,2.0 \mathrm{mM}$ of varying concentrations again suggested one electron transfer at microelectrode and a two-electron transfer process at macroelectrode (see SI section 4), consistent with the proposed mechanism.

\subsection{Electrochemical Detection of Piperine}

In this section, first, we report the analytical responses of piperine at a glassy carbon (GC) macroelectrode using conventional linear sweep cyclic voltammetry and suggest a data analysis method. Second, we carried out an analogous study using semi-circular potential sweep voltammetry with an optimised potential window to obtain amplified peaks and a simplified, improved procedure of background correction. Finally, we report a one-step extraction method of black pepper and the electrochemical detection method of the piperine content in real samples using both cyclic voltammetry and semi-circular potential sweep voltammetry. 

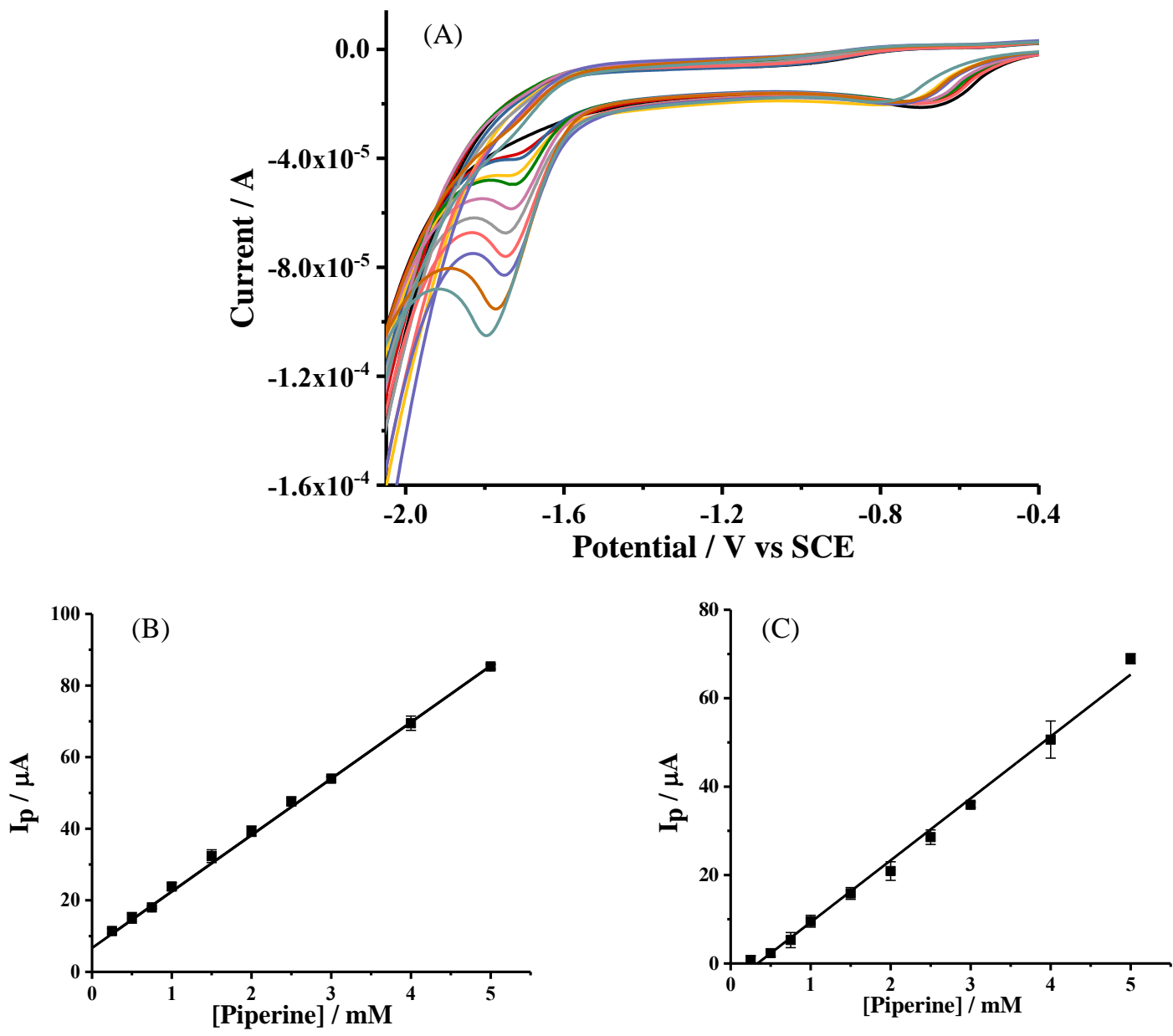

Figure 2. Detection of piperine at glassy carbon (GC) electrode in $60 \mathrm{EtOH}: 40 \mathrm{PBS}$ under air-saturated conditions using cyclic voltammetry: (A) Cyclic voltammetric responses of background and increasing concentrations from 0.25-5.0 mM. Scan rate: $100 \mathrm{mV} / \mathrm{s}$. (B) Calibration curve plot using the peak heights analysed by extrapolating the baseline. (C) Calibration curve plot using the current values read from the fixed potential of $-1.8 \mathrm{~V}$ after subtraction of background.

Having investigated the electrochemical behaviour of piperine in aqueous solution, we next turn to the detection of piperine in standard solutions and study the analytical responses of piperine at the glassy carbon (GC) macroelectrode. First, the cyclic voltammetric studies as described in section 3.1.1 were carried out under air-saturated conditions and likewise, the number of electrons transferred was calculated using the measured peak currents $\mathrm{I}_{\text {Expt }}$ and the calculated peak currents $\mathrm{I}_{\text {Irrev }}$ (see SI Section 5). Compared with the cyclic voltammetry measurements performed under degassed conditions, as 
expected, an additional, independent peak corresponding to oxygen reduction can be observed. Analysis at the piperine reduction showed that the number of electrons transferred was 2 and the diffusion coefficient determined from the slope of peak current vs. square root of scan rate plot via multiple electron-transfer, irreversible Randles-Sevcik equation was $5.0 \times 10^{-6} \mathrm{~cm}^{2} \mathrm{~s}^{-1}$, close to the values estimated under degassed condition $\left(4.8 \times 10^{-6} \mathrm{~cm}^{2} \mathrm{~s}^{-1}\right)$ and measured in chronoamperometry $\left(4.9 \times 10^{-}\right.$ $\left.{ }^{6} \mathrm{~cm}^{2} \mathrm{~s}^{-1}\right)$. The obtained results indicate the oxygen shows no interference with the peak current of the piperine reduction and the same electrochemical behaviour of piperine can be seen on GC macroelectrode regardless of the $60 \%$ ethanol $+40 \%$ PBS solution having degassed or not. Hence we conducted further measurements without degassing to allow a simpler and rapid detection of piperine.

Cyclic voltammetry was then conducted of piperine with the concentration in the range of $0.25-5.0$ $\mathrm{mM}$ in air-saturated $60 \%$ ethanol $+40 \%$ PBS. Three scans at each concentration were conducted and

the electrode was polished before each measurement. Figure 2(A) depicts the cyclic voltammetric response as a function of increasing concentration of piperine. The plot of peak height versus piperine concentration (Figure 2(B)) shows a linear relationship in the concentration range of $0.25-5.0 \mathrm{mM}$. The calibration curve is $\mathrm{I}_{\mathrm{p}} / \mu \mathrm{A}=(15.76 \pm 0.15)$ [piperine] $(\mathrm{mM})+(6.69 \pm 0.35)(\mu \mathrm{A}), \mathrm{N}=10, \mathrm{R}^{2}=0.999$. The peak height was analysed by the extrapolation of baseline chosen in the vicinity of the reductive peak. Note that the calibration curve plot in Figure 2(B) shows a not insignificant intercept in the y-axis making analysis unreliable.

A significant component to the background current is that resulting from the Faradaic reduction of the oxygen dissolved in the solution since as discussed above, it was chosen not to outgas this so as to give an overall simpler analytical procedure suitable to use by non-experts. Accordingly, we attempted to make a different and simpler background correction by simply measuring currents at a fixed potential on the voltammogram for all concentrations regardless of the potential of the peak current. This Figure 2(C) shows that if the current measured from a voltammogram recorded at $100 \mathrm{mVs}^{-1}$ and at a potential of $-1.80 \mathrm{~V}$ vs. SCE in absence of piperine is subtracted from the currents recorded under corresponding conditions in the presence of different concentrations of the analyte. Then a non-linear calibration curve 
results, this time with a positive $\mathrm{x}$-axis intercept. The limitations of the background corrections thus far attempted lie in the relatively low signal to noise ratio of the measurement. Accordingly, we turned to the use of semi-circular voltammetry.

\subsubsection{Using semi-circular potential sweep voltammetry for detection of piperine}

The recently proposed method to conduct potential sweep voltammetry using a semi-circular potential waveform for electroanalysis was found to show to a good agreement between theoretical predictions and experiments in the study of $\left[\mathrm{Ru}\left(\mathrm{NH}_{3}\right)_{6}\right]^{3+}{ }^{2+}$ redox couple at macroelectrode (Amin et al., 2019; Uchida et al., 2018). In particular, a notably higher current response can be achieved using this technique compared to that obtained from the conventional linear-sweep potential wave (Amin et al., 2019). In order to improve our analytical method of the detection of piperine and illustrate this new reported method for the first time in an analytical application, we explored the semi-circular potential sweep voltammetry of the piperine standard samples with the same concentrations at a glassy carbon (GC) electrode under air-saturated conditions.

In semi-circular potential sweep voltammetry, the potential of working electrode $E(t)$ follows a semicircular potential wave (Figure 3(A)):

$$
\begin{gathered}
E(t)= \pm A_{0} \sqrt{\left|1-\left[2\left(\frac{t}{t_{\max }}-c\right)\right]^{2}\right|}+E_{\text {centre }} \\
E_{\text {centre }}=\frac{E_{f}-E_{i}}{2}
\end{gathered}
$$

where $A_{0}$ is the amplitude of the potential wave, $t$ is the time and $t_{\max }$ is the duration of a single scan between the two potential limits. $\mathrm{c}=0$ for $t \leqslant t_{\max } / 2, \mathrm{c}=1$ for $t_{\max } / 2<t<t_{\max }$ and $E_{\text {centre }}$ is the midpoint of the applied potential window, which is experimentally defined relative to the reference electrode with the start potential $E_{i}$ and the vertex potential $E_{f}$. In contrast to the constant scan rate over the entire scan in linear sweep voltammetry, the scan rate resulting from the semi-circular wave shows significant variations with the sweep. As can be seen in Figure 3(A), the scan rate is close to zero at $t=0$ and $t=t_{\max }$ and increases transiently to infinite at $\mathrm{E}_{\text {centre. }}$. 

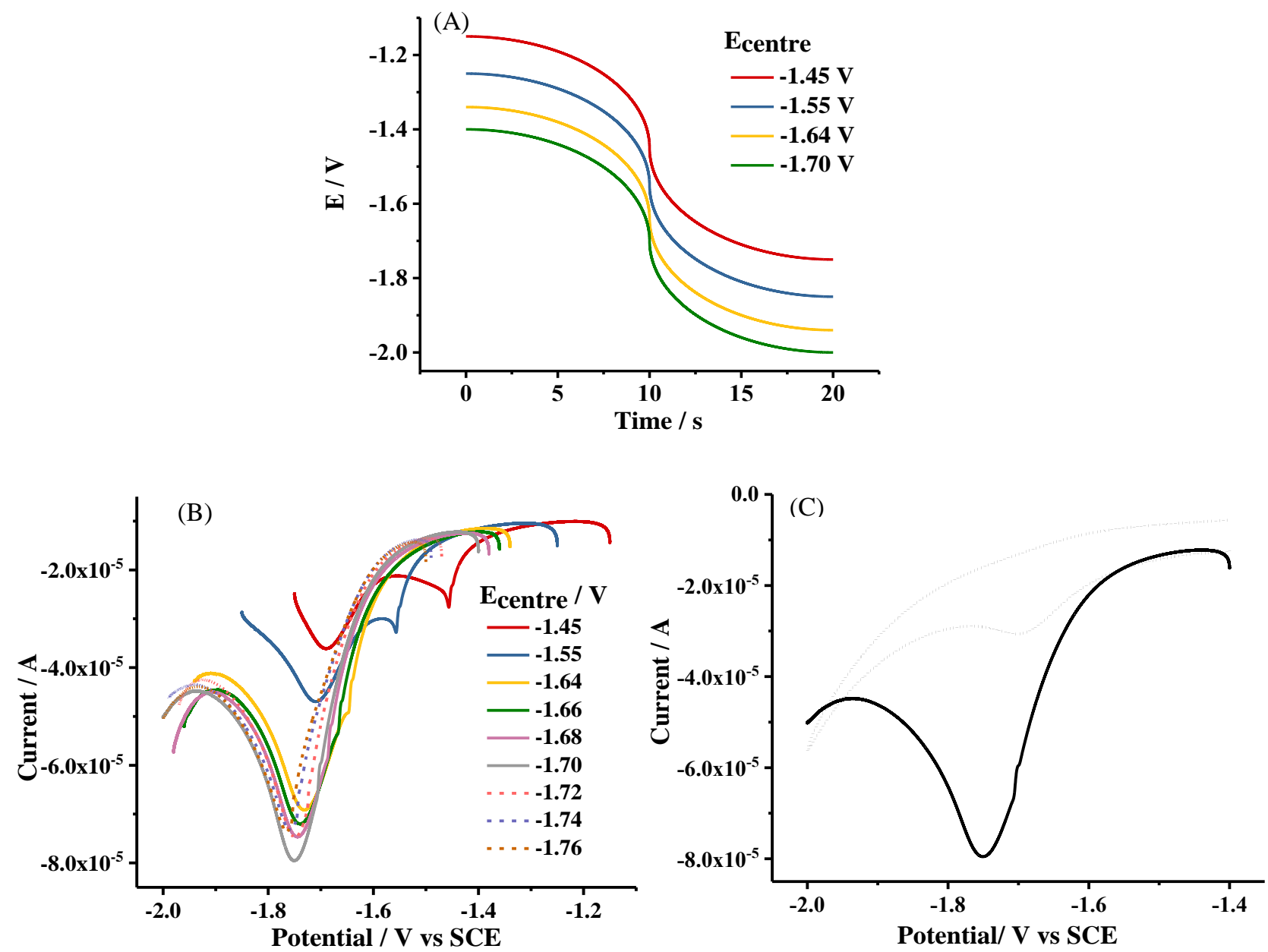

Figure 3. Origin and optimisation of semi-circular potential sweep voltammetry: (A) Semi-circular waveforms recorded for varying $\mathrm{E}_{\text {centre. }}$ Scan amplitude: $0.3 \mathrm{~V}$. (B) Semi-circular potential sweep voltammetric responses of $1.5 \mathrm{mM}$ of piperine at varying potential windows at scan amplitude of $0.3 \mathrm{~V}$ (solid lines) and $0.25 \mathrm{~V}$ (dash lines). (C) Semi-circular potential sweep voltammogram (solid line) at an average of scan rate of $30 \mathrm{mV} \mathrm{s}^{-1} \mathrm{vs}$. cyclic voltammogram (dot line) of $1.5 \mathrm{mM}$ piperine at $50 \mathrm{mV} \mathrm{s}^{-1}$ over a potential window: -1.4 to $-2.0 \mathrm{~V}$. Scan amplitude: $0.3 \mathrm{~V}$.

As discussed in previous work, by adjusting the position of $\mathrm{E}_{\text {centre }}$ to the close vicinity of the reduction potential of the studied target, the peak current reached a maximum value(Amin et al., 2019; Uchida et al., 2018). To determine the $\mathrm{E}_{\text {centre }}$ leading to the maximum peak current in our system, voltammetric measurements of $1.5 \mathrm{mM}$ piperine in $60 \%$ ethanol $+40 \%$ PBS using scan amplitudes of 0.3 and $0.25 \mathrm{~V}$ were carried out. It can be seen in Figure 3(B) when $E_{\text {centre }}=-1.70 \mathrm{~V}$ with scan amplitude as $0.3 \mathrm{~V}$, the peak current reached the maximum. Thus in further semi-circular potential sweep voltammetric experiments, the potential window of -1.40 to $-1.70 \mathrm{~V}$ was employed. Comparing with the cyclic voltammetry, we can see two main differences in semi-circular voltammetry. First, with the potential 


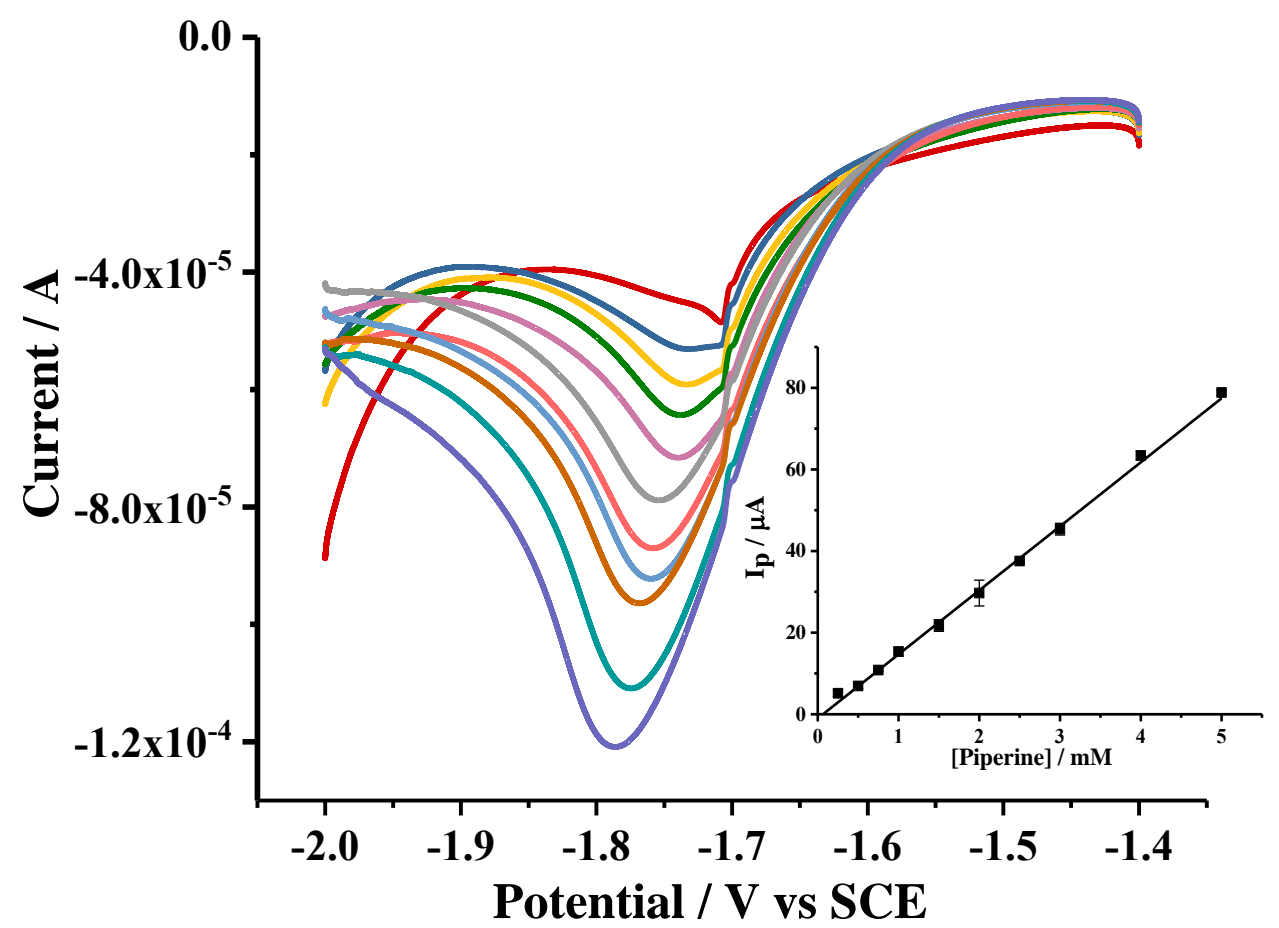

Figure 4. Semi-circular potential sweep voltammetric responses of the background and varying concentrations of piperine at glassy carbon (GC) electrode in $60 \mathrm{EtOH}$ : 40 PBS at air-saturated conditions. Scan rate: $50 \mathrm{mV} / \mathrm{s}$. Potential window: -1.40 to $-2.0 \mathrm{~V}$. Inset: Calibration curve plot using the current value at $-1.80 \mathrm{~V}$ after the subtraction of background.

window thus optimised, the obtained piperine reduction peak using semi-circular potential sweep voltammetry was significantly larger compared to the peak shown in the cyclic voltammetry at the same scan rate (Figure 3(C)). Second, in the semi-circular potential sweep voltammetry, (Figure 3(B) and (C)) kinks at the midpoint can be seen, arising from reaching the infinite scan rate.

Figure 4 depicts semi-circular potential sweep voltammetric responses for $0.25-5 \mathrm{mM}$ piperine in $60 \%$ ethanol $+40 \%$ PBS as a function of increasing concentrations of piperine. The sizes of the peaks in Figure 4 compare favourably with the signals shown in Figure 2(A). As discussed above, the obtained peak currents were substantially affected by the potential windows and values of $\mathrm{E}_{\text {centre. }}$ Consequently, current values at a fixed potential rather than the peak currents read at shifted potentials for varying concentrations were taken for calibration curve analysis. Several potentials near the peak potential were examined and $-1.8 \mathrm{~V}$ was chosen to be the suitable potential for further analysis (Full discussion see SI 
Section 6). For the current values at $-1.8 \mathrm{~V}$, a linear behaviour was found in the plot of these current values versus piperine concentration, in the concentration range of $0.25-5.0 \mathrm{mM}$. After subtracting the current from a single background scan at $-1.8 \mathrm{~V}$ vs. $\mathrm{SCE}$, the resulting calibration curve is $\mathrm{I}_{\mathrm{p}} / \mu \mathrm{A}=$ (15.69 \pm 0.23$)$ [piperine] $(\mathrm{mM})-(1.00 \pm 0.54)(\mu \mathrm{A}), \mathrm{N}=10, \mathrm{R}^{2}=0.999$ (Figure 4 inset).

The results provided the experimental validation for using semi-circular potential sweep voltammetry to study the analyte giving greatly amplified peaks. In the semi-circular potential sweep voltammogram, currents are read at a fixed potential and a simple background correction can be achieved via subtracting the background at the chosen potential, making the semi-circular potential sweep voltammetry a more advantageous method in data analysis than the conventional cyclic voltammetry.

\subsection{Analysis of a real sample}

In order to assess the feasibility and accuracy of electrochemistry for piperine analysis, we next looked at the detection of piperine in real samples. We first developed a procedure for the extraction of piperine from a black pepper sample purchased in a local supermarket. Among various methods of piperine isolation reported in the literature, the ultrasound-assisted extraction was found to be a rapid, simple, cheap method leading to high yield of piperine (Gorgani et al., 2017). Ethanol as a cheap, easy-handling solvent was suggested to give high extraction yields along with insonation(Rathod \& Rathod, 2014). Therefore, we chose ethanol as the extraction solvent and carried out the extractions with different sonication time periods. The preparation of samples was fully described in section 2.3. Ethanol was added to the vials with ground samples in a fixed ratio ( $5 \mathrm{~mL}$ ethanol to $0.5 \mathrm{~g}$ black pepper), which was then placed in an ultrasonic bath for the sonication times of 5, 10, 15 minutes. Experiments maximising the piperine content by using a long time sonication of 1 hour sonication followed by 1 day extraction were also carried out to obtain the maximised piperine contents. After sonication/extraction, the supernatant was mixed with PBS in the ratio of 60:40 and diluted with 60\% ethanol $+40 \%$ PBS. Cyclic voltammetric measurements at a glassy carbon (GC) electrode were then conducted. The results of extracts from three separate black pepper samples were recorded for each sonication time, as shown in 
Figure 5(A), where the results have been expressed in piperine content $(\% \mathrm{w} / \mathrm{w})$ of the original sample.

393 The resulted maximum piperine contents obtained at 1 hour sonication followed by 1 day extraction were found to be $(3.66 \pm 0.02) \% \mathrm{w} / \mathrm{w}$, in accordance with the values reported by literatures where the piperine content in fruit of $P$. nigrum quantified by HPLC technique was found to lie in the range of 3\%-6\% (w/w) (Mukherjee, P.K., 2002, Vasavirama, K. \& Upender, M., 2014). For the short time extractions, the results lie in the range of $(2.03 \pm 0.19) \% \mathrm{w} / \mathrm{w}$ to $(2.27 \pm 0.17) \% \mathrm{w} / \mathrm{w}$, which is approaching the maximum value at long time extractions. The short time extraction results show no evident growth in extraction efficiency with the increasing sonication time. It can be inferred the maximum extraction for a short time period had been achieved at some point before 5 minutes of sonication or without the assistance of sonication. In order to simplify the procedure and cut down the waiting time, we carried out the extraction by simply shaking the extract for 1 minute prior to leaving
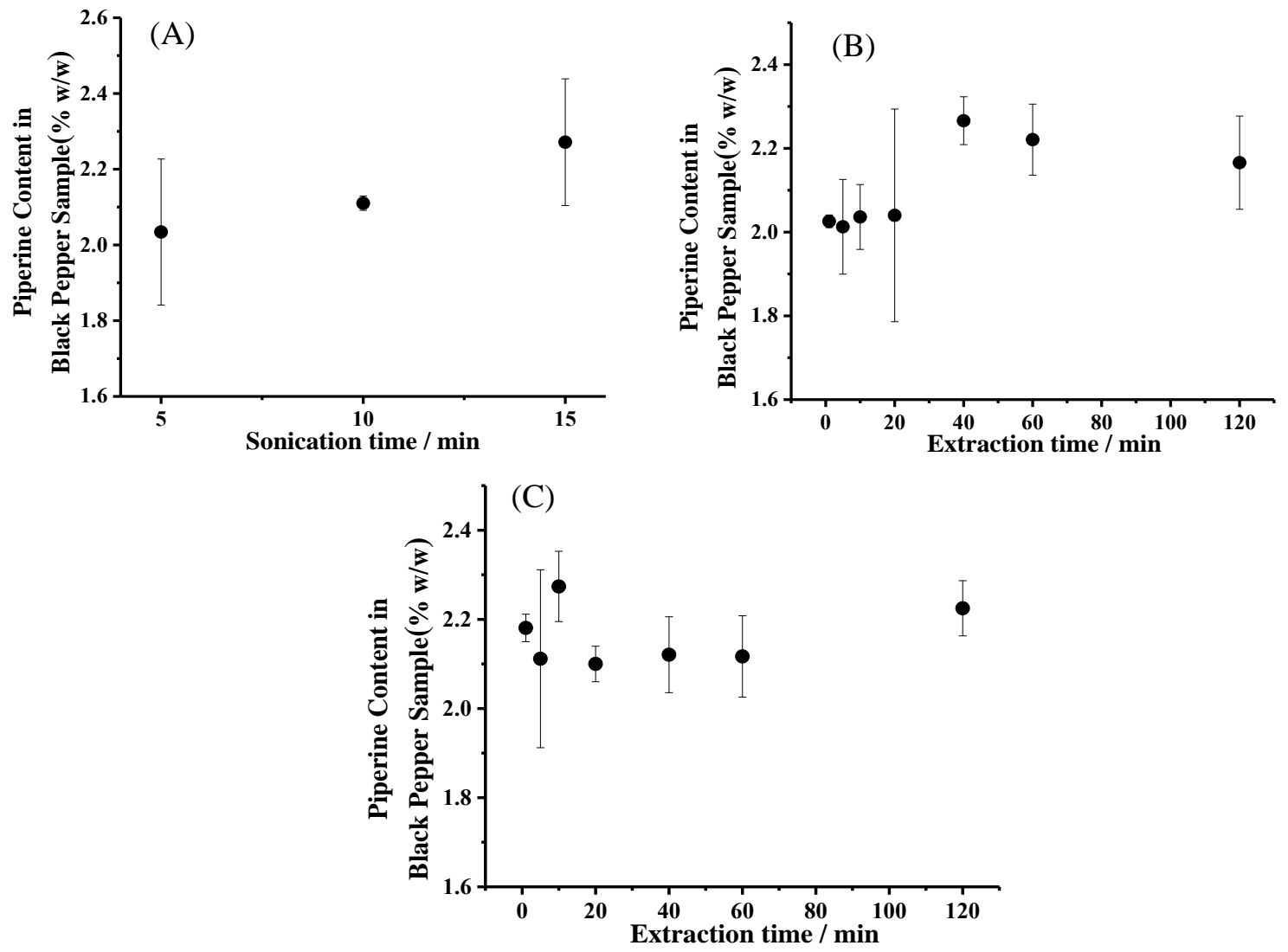

Figure 5. Piperine content detected in black pepper extracts at different extraction times by cyclic voltammetry using Figure 2(B) calibration curve (A) With sonication from 5 to 15 minutes (B) Without sonication from 0 to 

120 minutes. the extract to settle for 1-120 minutes. The rest of the procedure was used just as the procedure above with sonication. The results (Figure 5(B)) from the simplified procedure showed the piperine contents were found to be $(2.01 \pm 0.11) \% \mathrm{w} / \mathrm{w}$ to $(2.27 \pm 0.06) \% \mathrm{w} / \mathrm{w}$, which were approximately in the same range as the sonication method, suggesting the extraction for a short time period was essentially completed in one minute without sonication. The extracted sample was also measured after the addition of ethanol without shaking or settling, the piperine content was found to be ca. $(1.79 \pm 0.06) \% \mathrm{w} / \mathrm{w}$, just slightly below the range. From the results, it can be seen the extraction of piperine in black pepper is a rather fast process given that the black pepper had been ground into small particles, and our simplified procedure with any settling time from 1 to 120 minutes can be used for further quantification. For practical analysis our approach offers a good compromise with rapid analysis plus a reasonable accuracy. 


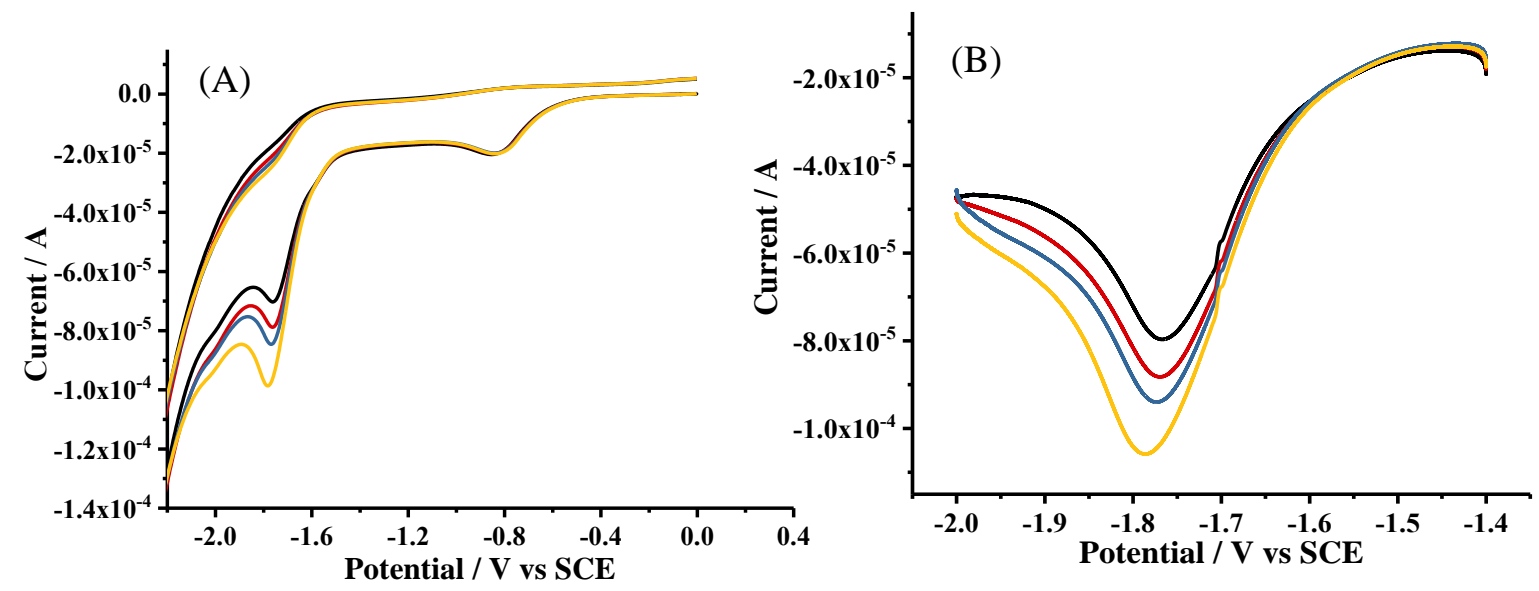

(C)

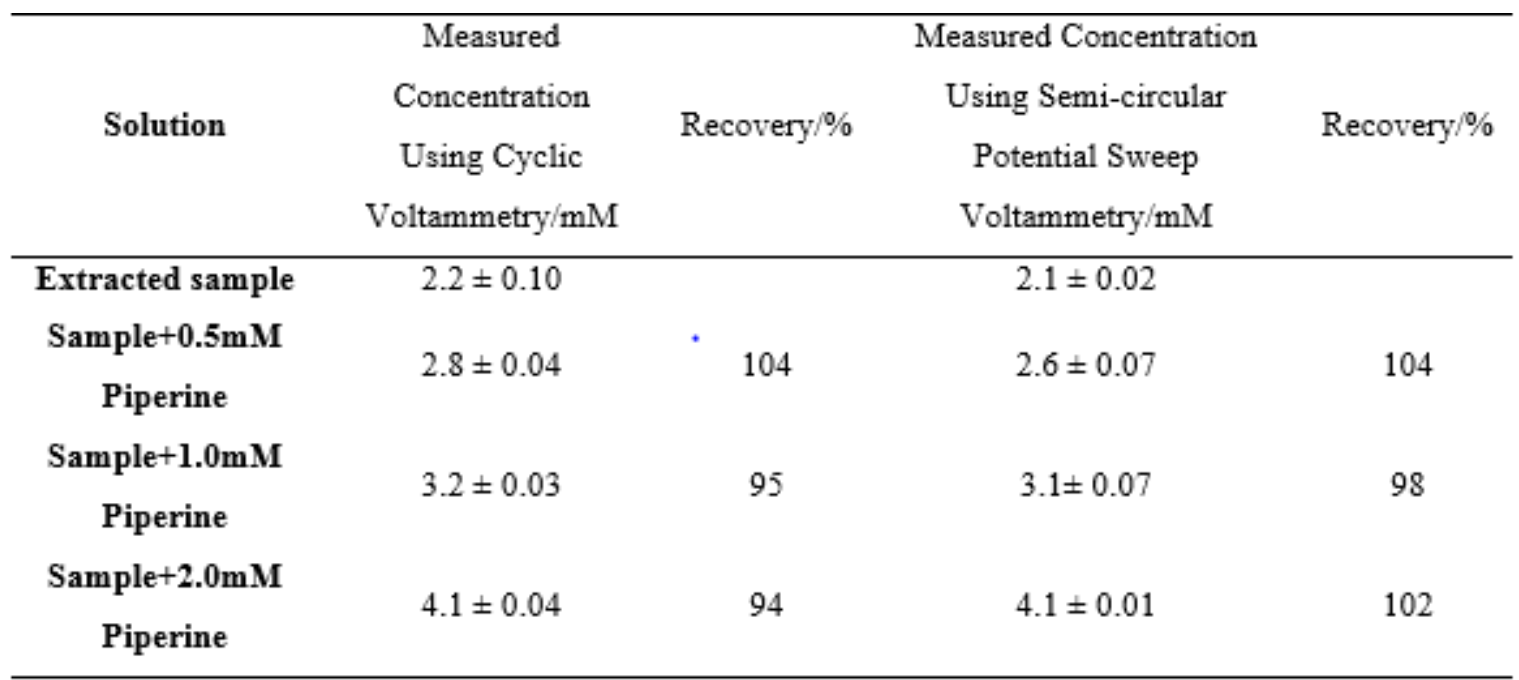

Figure 6. Voltammograms of real samples and the recovery experiment: (A) cyclic voltammograms of real sample extract (black line) extracts added with $0.5 \mathrm{mM}$ (red line), $1 \mathrm{mM}$ (blue line), $2 \mathrm{mM}$ (yellow line) standard piperine solutions. Scan rate: $100 \mathrm{mV} \mathrm{s}^{-1}(\mathrm{~B})$ semi-circular potential sweep voltammograms of real sample extract (black line) extracts added with $0.5 \mathrm{mM}$ (red line), $1 \mathrm{mM}$ (blue line), $2 \mathrm{mM}$ (yellow line) standard piperine solutions. The average of scan rate: $30 \mathrm{mV} \mathrm{s}^{-1}(\mathrm{C})$ Concentrations analysed by calibration curves in insets of Figure 2(B) and Figure 4 of detected piperine in extract and extracts with standard solutions and the corresponding recoveries.

Having developed the extraction procedure, we next employed the semi-circular potential sweep voltammetry for the quantification of piperine in real samples. The same simplified extraction procedure as above was carried out with a settling time of 1-120 minutes using the semi-circular potential sweep voltammetric method. The piperine contents depicted in Figure 5(C) lie in the range of $(2.10 \pm 0.03) \%$ $\mathrm{w} / \mathrm{w}-(2.27 \pm 0.08) \% \mathrm{w} / \mathrm{w}$, which was in accordance with the result obtained from using cyclic voltammetry. 
The accuracy of the extraction procedure was validated by a "recovery" test (Carr \& Wahlich, 1990), where known concentration standard solutions were used to "spike" diluted extracts prior to voltammetric measurements (Figure 6(A)(B)). The percentage recoveries were evaluated by both cyclic voltammetric and the semi-circular potential sweep voltammetric methods. The peak heights measured in cyclic voltammograms and semi-circular potential sweep voltammograms are converted to the concentrations of piperine using the calibration curves in Figure 2(B) and Figure 4. Values close to $100 \%$ were attained in both methods (Figure 6(C)).

\section{Conclusions}

Cyclic voltammetry and semi-circular potential sweep voltammetry have both been successfully applied to the detection and quantification of piperine in standard solutions and real samples using a glassy carbon (GC) macroelectrode. In particular, the employment of semi-circular potential sweep voltammetry provides the advantage of amplifying peaks to facilitate the electrochemical determination of species otherwise showing low sensitivity. To the best of our knowledge, this the first time semicircular potential sweep voltammetry has been applied for electroanalytical detection. The electrochemical reduction mechanism of piperine was investigated utilizing a combination of cyclic voltammetry and potential step chronoamperometry and a possible mechanism was inferred. This proposed method of detection may become useful for application in pharmaceutical production and the food industry, serving as a facile and inexpensive substitute for HPLC and GC-MS, etc..

\section{Conflict of interests}

The authors declare that they have no conflict of interest. 
Amin, H. M. A., Uchida, Y., Kätelhön, E., \& Compton, R. G. (2019). Semi-circular potential sweep voltammetry: Experimental verification and determination of the formal potential of a reversible redox couple. Journal of Electroanalytical Chemistry, 836, 62-67. https://doi.org/10.1016/j.jelechem.2019.01.058

Balakrishnan, V., Varma, S., \& Chatterji, D. (2001). Piperine augments transcription inhibitory activity of rifampicin by severalfold in Mycobacterium smegmatis. Current Science, 80(10), 1302-1305. https://doi.org/10.2307/24105044

Banks, C. E., Evans, R. G., Rodrigues, J., Turner, P. G., Donohoe, T. J., \& Compton, R. G. (2005). Cryoelectrochemistry: electrochemical reduction of 2(RS)-methyl-1-(tert-butoxycarbonyl)-2iodomethyl-2,5-dihydropyrrole- 2-carboxylate. Tetrahedron, 61, 2365-2372. https://doi.org/10.1016/j.tet.2005.01.022

Bard, A. J., \& Faulkner, L. R. (2001). Electrochemical methods : fundamentals and applications $\left(2^{\text {nd }}\right.$ ed.). New Jersey: Wiley.

Batchelor-Mcauley, C., Ellison, J., Tschulik, K., Hurst, P. L., Boldt, R., \& Compton, R. G. (2015). In situ nanoparticle sizing with zeptomole sensitivity. Analyst, 140(15), 5048. https://doi.org/10.1039/c5an00474h

Carr, G. P., \& Wahlich, J. C. (1990). A practical approach to method validation in pharmaceutical analysis. Journal of Pharmaceutical \& Biomedical Analysis, 8(8-12). 613-618. https://doi.org/10.1016/0731-7085(90)80090-C

Compton, R. G., \& Banks, C. E. (2018). Understanding Voltammetry ( $3^{\text {rd }}$ ed.). Singapore: World Scientific.

da Silva, A. P., Mota, S. D. C., Bieber, L. W., \& Navarro, M. (2006). Homogeneous electro-mediated reduction of unsaturated compounds using $\mathrm{Ni}$ and $\mathrm{Fe}$ as mediators in DMF. Tetrahedron, 62(23), 
498

499

500

501

502

503

504

505

506

507

508

509

510

511

512

513

514

515

516

517

518

519

520

Shoup, D., \& Szabo, A. (1982). Chronoamperometric current at finite disk electrode. Journal of Electroanalytical Chemistry, 140(2), 237-245. https://doi.org/10.1016/0022-0728(82)85171-1

Doucette, C. D., Hilchie, A. L., Liwski, R., \& Hoskin, D. W. (2013). Piperine, a dietary phytochemical, inhibits angiogenesis. The Journal of Nutritional Biochemistry, 24(1), 231-239. https://doi.org/10.1016/j.jnutbio.2012.05.009

Gallagher, R., Shimmon, R., \& McDonagh, A. M. (2012). Synthesis and impurity profiling of MDMA prepared from commonly available starting materials. Forensic Science International, 223(1-3), 306-313. https://doi.org/10.1016/j.forsciint.2012.10.006

Gorgani, L., Mohammadi, M., Najafpour, G. D., \& Nikzad, M. (2017). Piperine-The Bioactive Compound of Black Pepper: From Isolation to Medicinal Formulations. Comprehensive Reviews in Food Science and Food Safety, 16(1), 124-140. https://doi.org/10.1111/1541-4337.12246

Hirasa, K., \& Takemasa, M. (1998). Spice science and technology. Boca Raton Fla: CRC Press.

Jain, R., \& Verma, A. (2017). Voltammetric Quantification of Phytoesterone 1-[5-(1, 3- Benzodioxol5-yl)-1-oxo-2, 4-pentadienyl] Piperidine. International Journal of Electrochemical Science, 12, 3459-3471. https://doi.org/10.20964/2017.04.29

Janakiraman, K., \& Manavalan, R. (2008). Studies on Effect of Piperine on Oral Bioavailability of Ampicillin and Norfloxacin. African Journal of Traditional, Complementary, and Alternative Medicines, 5(3), 257.

Ju, M. J., Hayama, K., Hayashi, K., \& Toko, K. (2003). Discrimination of pungent-tasting substances using surface-polarity controlled sensor with indirect in situ modification. Sensors and Actuators, B: Chemical, 89(1-2), 150-157. https://doi.org/10.1016/S0925-4005(02)00457-4

Kim, J. S., Oh, D. H., Choi, H. M., Sur, B., Lim, S., Kim, J. Y., .. \& Kim, K. S. (2009). Piperine inhibits eosinophil infiltration and airway hyperresponsiveness by suppressing T cell activity 
and Th2 cytokine production in the ovalbumin-induced asthma model. Journal of Pharmacy and Pharmacology, 61(3), 353-359. https://doi.org/10.1211/jpp.61.03.0010

Mao, Q., Huang, Z., Zhong, X., Xian, Y., \& Ip, S. (2014). Piperine reverses the effects of corticosterone on behavior and hippocampal BDNF expression in mice. Neurochemistry International, 74, 36-41. https://doi.org/10.1016/j.neuint.2014.04.017

Mueller, K., \& Hingst, J. (2013). The athlete's guide to sports supplements. Leeds, United Kingdom: Human Kinetics.

Mukherjee, P. K. (2002). Quality Control and Evaluation of Herbal Drugs : Evaluating Natural Products and Traditional Medicine. India: Business Horizons

Noyer, I., Fayet, B., Pouliquen-Sonaglia, I., Guerere, M., \& Lesgard, J. (1999). Quantitative analysis of pungent principles of pepper oleoresins: Comparative study of three analytical methods. Analusis, 27, 69-74. https://doi.org/10.1051/analusis:1999109

Paddon, C. A., Silvester, D. S., Bhatti, F. L., Donohoe, T. J., \& Compton, R. G. (2007). Coulometry on the Voltammetric Timescale: Microdisk Potential-Step Chronoamperometry in Aprotic Solvents Reliably Measures the Number of Electrons Transferred in an Electrode Process Simultaneously with the Diffusion Coefficients of the Electroactive Spec. Electroanalysis, 19(1), 11-22. https://doi.org/10.1002/elan.200603667

Parthasarathy, V., Chempakam, B., \& Zachariah, J. (2008). Chemistry of Spices. London: CABI.

Pedersen, M. E., Metzler, B., Stafford, G. I., Van Staden, J., Jäger, A. K., \& Rasmussen, H. B. (2009). Amides from Piper capense with CNS activity - A preliminary SAR analysis. Molecules, 14(9), 3833-3843. https://doi.org/10.3390/molecules 14093833

Peter, K. V. (2001). Handbook of herbs and spices. Cambridge, United Kingdom: Woodhead Publishing Limited and CRC Press.

Rathod, S. S., \& Rathod, V. K. (2014). Extraction of piperine from Piper longum using ultrasound. 
Sabina, E. P., Souriyan, A. D. H., Jackline, D., \& Rasool, M. K. (2010). Piperine, an active ingredient of black pepper attenuates acetaminophen-induced hepatotoxicity in mice. Asian Pacific

Shimada, C., Uesawa, Y., Ishihara, M., Kagaya, H., Kanamoto, T., Terakubo, S., ... Sakagami, H. (2014). Quantitative structure-cytotoxicity relationship of piperic acid amides. Anticancer

Silvester, D. S., Wain, A. J., Aldous, L., Hardacre, C., \& Compton, R. G. (2006). Electrochemical

Vasavirama, K., \&Upender, M., 2014. Piperine: a valuable alkaloid from piper species. Int.

Sunila, E., \& Kuttan, G. (2004). Immunomodulatory and antitumor activity of Piper longum Linn. and piperine. Journal of Ethnopharmacology, 90(2-3), 339-346. https://doi.org/10.1016/j.jep.2003.10.016

Ternes, W., \& Krause, E. L. (2002). Characterization and determination of piperine and piperine isomers in eggs. Analytical and Bioanalytical Chemistry, 374(1), 155-160. https://doi.org/10.1007/s00216-002-1416-6

Uchida, Y., Kätelhön, E., \& Compton, R. G. (2018). Linear sweep voltammetry with non-triangular waveforms: New opportunities in electroanalysis. Journal of Electroanalytical Chemistry, 818, 140-148. https://doi.org/10.1016/j.jelechem.2018.04.028 

nigrum L.) and piperine in rats with high fat diet induced oxidative stress. Redox Report Communications in Free Radical Research, 9, 105-110. https://doi.org/10.1179/135100004225004742

Wang, Y., Limon-Petersen, J. G., \& Compton, R. G. (2011). Measurement of the diffusion coefficients of $\left[\mathrm{Ru}\left(\mathrm{NH}_{3}\right)_{6}\right]^{3+}$ and $\left[\mathrm{Ru}\left(\mathrm{NH}_{3}\right)_{6}\right]^{2+}$ in aqueous solution using microelectrode double potential step chronoamperometry. Journal of Electroanalytical Chemistry, 652, 13-17. https://doi.org/10.1016/j.jelechem.2010.12.011

Xiong, L., Aldous, L., Henstridge, M. C., \& Compton, R. G. (2012). Investigation of the optimal transient times for chronoamperometric analysis of diffusion coefficients and concentrations in non-aqueous solvents and ionic liquids. Analytical Methods, 4(2), 371-376. https://doi.org/10.1039/c1ay05667k

Zarai, Z., Boujelbene, E., Ben Salem, N., Gargouri, Y., \& Sayari, A. (2013). Antioxidant and antimicrobial activities of various solvent extracts, piperine and piperic acid from Piper nigrum. LWT - Food Science and Technology, 50(2), 634-641. https://doi.org/10.1016/j.lwt.2012.07.036 